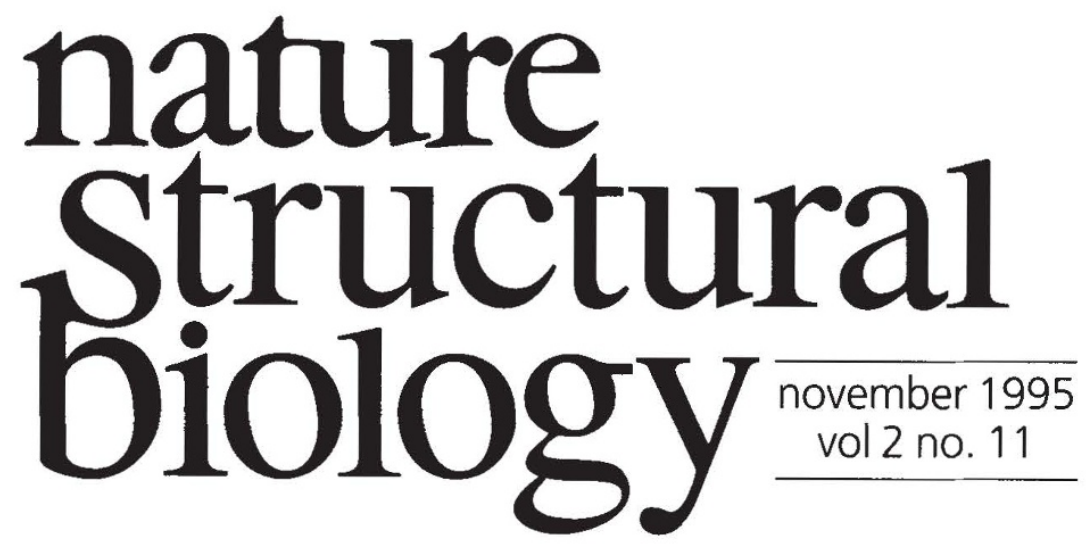

\title{
Meetings and membrane proteins
}

Heidelberg, Germany - A four-day meeting entitled Structure and function of proteins must inevitably be a disappointment to some attendees. After all, the sweep of topics that could be included under such a banner is vast and, of course, impossible to accommodate in such a short time. Nonetheless, a recent meeting ${ }^{*}$ took just such an ambitious title as its theme and managedwith considerable success-to begin weaving together some of the more important issues that confront researchers interested in the inner workings of proteins.

The recent determination of a number of structures of membrane proteins provided one of the strongest threads running through the meeting. This was perhaps unsurprising at such an ostensibly European conference given that the study of membrane protein structure has been — and still is - dominated by scientists working in Europe; a state of affairs that is, to say the least, lamentable from the point of view of the biological research community in the USA.

For structures of paid-up membrane proteins, one had to look to the European presentations. And these provided a veritable feast: the bacterial light harvesting protein (Andy Freer, Glasgow, UK); the plant light-harvesting complex, LHC-II (Werner Kühlbrandt, Heidelberg, Germany); bacteriorhodopsin and the mechanism by which it pumps protons across the membrane (Richard Henderson, Cambridge, UK); the open form of the acetylcholine receptor (Nigel Unwin, Cambridge, UK); and, of course, cytochrome $c$ oxidase (Hartmut Michel, Frankfurt, Germany: for further discussion of this enzyme see ref. 1, this issue).

While the problems associated with determining the structures of membrane proteins are formidable (but certainly not insurmountable) and often require 10-20 years of effort, the vital role that they play in biology clearly makes understanding their structure and function a top priority in biomedical research. The success of the scientists in Europe can, for the most part, be directly attributed to the long-term funding policies of a number of government granting agencies and pre-eminent research institutes; the Medical Research Council's Laboratory of Molecular Biology in the UK, the Biozen-

*Structure and function of proteins, 25-28 September, 21 st European Molecular Biology Organisation (EMBO) Symposium, European Molecular Biology Laboratory, Heidelberg, Germany. trum in Basel, Switzerland, and the system of Max Planck Institutes in Germany, for example.

The USA, on the other hand, operates under a system of meritocracy that is so demanding of results in the short term (the 'publish or perish' syndrome in extremis) that the study of membrane proteins is effectively stifled. It is clear that the mechanisms for funding research in this area are both inadequate and 


\section{IMAGE UNAVAILABLE FOR COPYRIGHT REASONS}

Clearly public demand (and money) in the United States can drive important and risky research (image from ref. 2). inappropriate. Indeed, the two presentations from US laboratories in the 'protein-membrane interactions' session did not directly deal with proteins in membranes, as Wim Hol (Seattle, Washington, USA) made clear in reporting his research on the soluble forms of members of the cholera toxin family.

The (some may say) short-sighted funding strategies in the United States are happily not sufficient to suppress all risky and ambitious research. Robert Griffin (Cambridge, Massachusetts, USA), the other presentation from the US in the session, described an impressive multidisciplinary analysis of the structure of a fragment of $\beta$-amyloid, the molecular species found in Alzheimer's disease plaques (see this issue of Nature Structural Biology ${ }^{2,3}$ ). Clearly, public demand (and money) in the United States can sometimes drive important, ground-breaking-and high risk-research that has broader implications for basic science.

Long term support is not the only factor in successfully determining membrane protein structure. Personal crusades by a few individual scientists, who have often spent years struggling to characterize, purify and crystallize a particular membrane protein, have also played an important part in moving the field forward despite the funding problems. But again, such crusades have been by mainly European scientists: for example, Richard Cogdell's work on the bacterial light harvesting protein, and Wolfram Welte's work on porins. Indeed, the porins also featured at the meeting and illustrated the mechanistic details that membrane protein research can and will yield.

Found primarily in the outer membrane of Gram-negative bacteria, porins function to facilitate the diffusion of nutrient solutes across the membrane. They are constructed from 16- or 18-stranded antiparallel $\beta$-barrels that have a pore at their centre. The structure of the complex between a malto-porin and its substrate, maltose (Georg Schultz, Freiburg, Germany) suggests an elegant mechanism by which the oligosaccharide is threaded through the channel. The pore is lined with three tryptophan and three tyrosine residues, providing a hydrophobic surface with which the sugar rings of the substrate can interact. The spacing between the hydrophobic residues down the channel is slightly wider than that of the glucose units in the oligosaccharide, so the two are only in register at every sixth sugar residue. This would preclude extensive interaction between pore and substrate, thus preventing the channel from becoming blocked with tightly bound sugar. The diameter of the channel is just wide enough to accommodate a glucose unit, and selectivity for this monosaccharide unit may be determined by a 'nick' in the throat of the channel which could accommodate passage of the $6{ }^{\prime} \mathrm{OH}$.

The only grumbles, and there were a few, concerned the heavy emphasis of the meeting on the description of structures determined by X-ray crystallography. Nuclear magnetic resonance (NMR) spectroscopy was poorly represented, giving its increasing importance in the study of structural biology. Indeed, there was some (good-natured) snipping between the NMRists and crystallographers, with Kurt Wüthrich and others coming to the defence of the younger technology. Also lacking were studies that more directly addressed function: insight into the actions of proteins were for the most part limited to those revealed by the structure. This last may be more a reflection of the still youthful nature field, which as it matures will naturally become more functionally oriented. These points aside, the meeting was a thumping success and will-perhaps-encourage the $\mathrm{EMBO}$ to continue to hold such annual symposia.

1. Adman, E.T. Nature struct. Bio/ 2, 929-931 (1995)

2. Lansbury, P. et al. Nature struct. Biol 2, 990-998(1995).

3. Mattson, M.P. Nature struct. Biol 2, 926-928 (1995).

4. Ostermeier, C., Iwata, S. Ludwig, B. \& Michel, H. Nature struct. Biol. 2, 842-846 (1995) 